\title{
Experimental Studies for the characterization of the mixing processes in negative buoyant jets
}

\author{
L.A. Besalduch ${ }^{1, \mathrm{a}}$, M.G. Badas ${ }^{1}$, S. Ferrari ${ }^{1}$, and G. Querzoli ${ }^{1}$ \\ ${ }^{1}$ University of Cagliari, DICAAR (Dipartimento di Ingegneria Civile, Ambientale e Architettura), 09123 Cagliari, Italy
}

\begin{abstract}
A negatively buoyant jet (NBJ) corresponds to the physical phenomenon that develops when a fluid is discharged upwards into a lighter environment or downwards into a heavier receptor fluid. In a NBJ the flow is initially driven mostly by the momentum, so it basically behaves as a simple jet released with the same angle, while far from the outlet the buoyancy prevails, bending the jet axis down and making it similar to a plume. The coexistence in the same phenomenon of both the characteristics of simple jets and plumes makes the NBJs a phenomenon still not entirely explained but, considering also the numerous practical applications, very interesting to study. Here some of the experimental results are presented. The laboratory experiment were obtained on a model simulating a typical sea discharge of brine from desalination plants: a pipe laid down on the sea bottom, with orifices on its lateral wall, releasing brine (heavier than the sea water) with a certain angle to the horizontal, in order to increase the jet path before sinking to the seafloor. A non-intrusive image analysis technique, namely Feature Tracking Velocimetry, is applied to measure velocity fields, with the aim at understanding the influence of some non-dimensional parameters driving the phenomenon (e.g. Reynolds number, release angle) on the structure of the NBJ and of the turbulence.
\end{abstract}

\section{Introduction}

A negatively buoyant jet (NBJ), is the physical phenomenon that develops when a fluid is discharged upwards into a lighter environmental receptor or downwards into a heavier receptor fluid. NBJs have many practical applications: for example: sea discharge of brine from desalination plants through submerged outfalls, e.g. Ferrari et al 2010 [1], Cipollina et al. 2005 [2], or Kikkert et al. 2007 [3]), oil or gas drilling facilities leaching of mineral salts domes (McLellan and Randall 1986 [4]), sea discharges of effluents from wastewater treatment plants (Koh and Brooks 1975 [5]), ventilation of aircraft hangars, heated using ceiling-mounted fans (e.g. Baines et al. 1989 [6]), the improvement of water quality by forced mixing in reservoirs and harbors (e.g. McClimans et al. 2000 [7]), vehicle exhausts and accidental leaks of hazardous gases (Lane-Serff 1993 [8]), the replenishment of magma chambers in the Earth's crust (Turner and Campbell 1986 [9]; Campbell and Turner 1989 [10]), explosive volcanic jets (Kaminski and Carazzo 2004 [11]), etc.

Many studies were dedicated to vertical NBJs (e.g. Goldman and Jaluria 1986 [12], Campbell and Turner 1986 [9], Baines et al. 1990 [13], Zhang et al 1998 [14], Davidson et al. 2002 [15], Kaye et al. 2007 [16]), and to inclined NBJs. The first one was possibly Bosanquet et al. (1961) [17] and presented results for horizontal and $45^{\circ}$ jets. Later, Zeitoun et al (1970) [18] studied $30^{\circ}, 45^{\circ}$ and $60^{\circ}$ inclined jets and they obtained that the maximum path and therefore the maximum dilution was found for angles of $60^{\circ}$. Roberts and Tom (1987) [19] released the jet upward vertically or with an angle of $60^{\circ}$ to the horizontal, finding that the maximum height of the $60^{\circ}$ jet was smaller than of vertical jet. Later Baines et al (1990) [13] found that the largest maximum height is for angles of $83^{\circ}$. In contrast Lane-Serff et al (1993) [8] for angles between $0^{\circ}$ and $75^{\circ}$, found that the maximum height increases with the angle. Roberts et al. (1997) [20] studied jets with an angle of $60^{\circ}$ focusing on the impact point and the bottom layer dilution. Bloomfield and Kerr (2002) [21] focused on the maximum edge heights for jets with inclination between $30^{\circ}$ and $90^{\circ}$. Cipollina et al. (2005) [2] studied angles of $30^{\circ}, 45^{\circ}$ and $60^{\circ}$ with different Froude number, focusing on the maximum height, distance from the source and dilution of the impact point. Kikkert et al. (2007 [22], 2010 [23]) studied different horizontal and vertical inclination of the release, and of the ambient receptor, measuring dilution and trajectories. Papakostantis et al. (2011a [24], 2011b [25]) focusing on inclination between $45^{\circ}$ to $90^{\circ}$, at different Froude number, measured among others, the turbulent

${ }^{\mathrm{a}}$ besalduch@unica.it 
concentration fluctuation $\left(\mathrm{C}_{\mathrm{rms}}\right)$ and the geometrical characteristics of the jet.

In contrast with the previous quoted authors, that discharged the jet at the end of a pipe, Ferrari and Querzoli (2004 [26], 2010 [1]) studied NBJs emitted from a sharp edged orifice, as this kind of release allows a larger entrainment (Mi et al. 2001 [27], 2007 [28]); the inclination was from $45^{\circ}$ to $90^{\circ}$, and these works explain the non-axisymmetry of NBJs, due to the different stratifications in the upper and lower boundary of a NBJ (see also Ferrari and Querzoli, ISSF 2011 [29]). Moreover, they showed that the maximum height of the jet axis increases from $45^{\circ}$ to approximately $80^{\circ}$, in agreement with the results of Bloomfield and Kerr (2002) [21], to then decrease, because of the re-entrainment phenomenon.

These works, were generally, performed by means of optical techniques, such as Light Attenuation (LA) or Light Induced Fluorescence (LIF), so the only measured quantities were the concentration fields; only very few investigations were conducted using Particles Image Velocimetry (PIV) techniques to study the velocity fields. Cenedese et al. 2005 [30] used both LIF and Particle Tracking Velocimetry (PTV) techniques, to study velocity and concentration profiles at different Froude numbers for horizontal jets. A paper based on PIV measures for inclined NBJs, released at the end of a pipe, is the one by Lai and Lee (2012) [31]: the release angles were between $15^{\circ}$ and $60^{\circ}$ and, the authors compared their results with two previous work of Shao and Law (2010 [32], 2011 [33]), with inclination of $30^{\circ}$ and $45^{\circ}$.

The main target of this work is to investigate the influence of the Reynolds number on some turbulent quantities that govern the mixing processes, via a novel Feature Tracking Velocimetry (FTV) technique.

\section{Dimensional analysis}

As previous stated, a negatively buoyant jet (NBJ) is a phenomenon that develops when a fluid is released upward into a lighter ambient receptor, or downwards into a heavier. In a NBJ, the quantities that govern the flow are, the initial flux of volume (Q), the initial flux of momentum (M) and the initial flux of buoyancy per unit mass (B) (List et al, 1979 [34]). For a round orifice, in a stagnant receptor these quantities are:

$$
\begin{gathered}
Q=\frac{1}{4} \pi D^{2} W \\
M=\frac{1}{4} \pi D^{2} W^{2} \\
B=g \frac{\Delta \rho_{0}}{\rho} Q=g^{\prime}{ }_{0} Q
\end{gathered}
$$

where $\mathrm{D}$ is the diameter of the outlet, $\mathrm{W}$ is the mean velocity at the outlet, $g$ is the gravitational acceleration, $\rho$ is the density of the receptor and $\bullet \rho_{0}$ is the difference in density between the discharged fluid and the surrounding fluid.

It is possible to define two independent length scales:

$$
\begin{aligned}
l_{Q} & =\frac{Q}{M^{1 / 2}} \\
l_{M} & =\frac{M^{3 / 4}}{B^{1 / 2}}
\end{aligned}
$$

The former, $\mathrm{l}_{\mathrm{Q}}$ gives a magnitude of the distance downstream from the jet orifice, at which all the properties of the jet will be a function of $Q$ and $M$, so it represents the order of magnitude of the influence of the initial geometry; the latter, $1_{\mathrm{M}}$, gives a magnitude of the distance where the buoyancy prevails on the initial momentum and the jet behaves principally like a plume. The ratio between these two length scales is proportional to the Froude Number (Fr):

$$
F r=\left(\frac{\pi}{4}\right)^{1 / 4} \cdot\left(\frac{l_{M}}{l_{Q}}\right)=\frac{W}{\sqrt{g \cdot \frac{\Delta \rho}{\rho} \cdot D}}
$$

The non-dimensional parameters that control the behavior of NBJs, apart from the Froude number, are the Reynolds number $\operatorname{Re}=W \cdot D / v$, where: $v$ is the kinematic viscosity of the discharged fluid, and $\theta$, the inclination to the horizontal, that control the misalignment between the buoyancy and the momentum.

\section{Experiments}

\subsection{Set-up}

The experimental set-up simulates a portion of a pipe laid down on the sea floor, which discharges the effluent from the orifices along the pipe wall. This configuration is typical of submarine outfalls (Wright et al. 1982 [35], Avanzini et al. 2006 [36]).

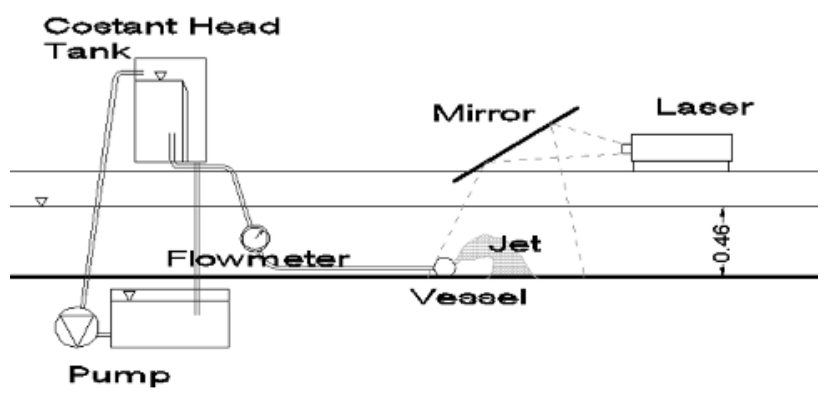

Fig. 1. Sketch of the experimental set-up.

This model (shown in figure 1) consists in a $21 \mathrm{~m}$ long and $30 \mathrm{~cm}$ wide flume, with glass walls, filled with $46 \mathrm{~cm}$ of water (enough to avoid an interaction between the jet and the free surface), at rest, to simulate a stagnant receiving body, and with a flat bottom, to have the shortest path available for the jet dilution. The discharge comes through a pipe, which is connected to a constant head tank, by means of a cylindrical vessel of $0.10 \mathrm{~m}$ diameter, with a sharp-edge orifice of diameter $\mathrm{D}=4$ $\mathrm{mm}$, on its lateral wall. The released fluid is a solution of water, sodium sulphate, to increase the density, and pollen particles (around $50 \mu \mathrm{m}$ in diameter), to perform 
experiments with a Feature Tracking Velocimetry (FTV) technique (see below chapter 3.3).

The jet middle vertical section is lighted, by a $1 \mathrm{~mm}$ tick light sheet, generated by a diode pumped green laser with a cylindrical lens. The phenomenon is recorded by a high speed video camera having a frequency of $400 \div 800$, and a maximum spatial resolution of $2280 \times 1728 \times 8$ bit.

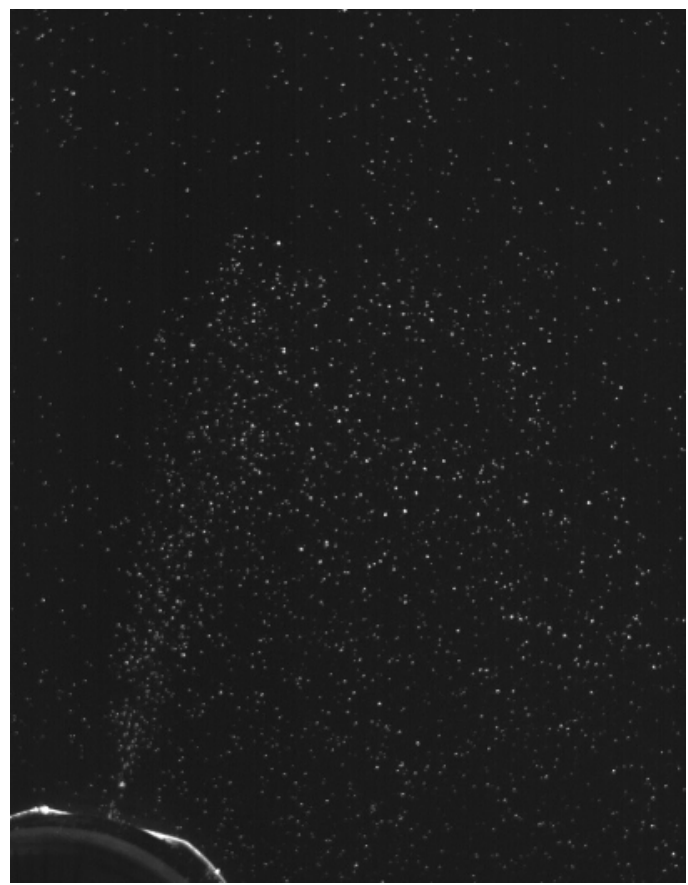

Fig. 2. A snapshot of a negatively buoyant jet, with a densimetric Froude number of 15 and a release angle on the horizontal of $80^{\circ}$, seeded with pollen particles for the FTV analysis.

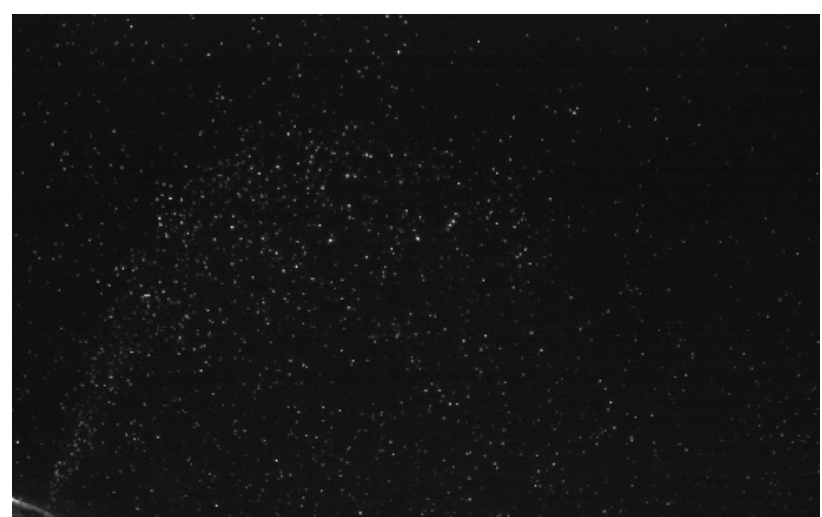

Fig. 3. A snapshot of a negatively buoyant jet, with a densimetric Froude number of 15 and a release angle on the horizontal of $65^{\circ}$.

\subsection{Experiments}

The experiments were performed with $\mathrm{Fr}=15$, release angle to the horizontal $\theta=65^{\circ}$ and $80^{\circ}$, and two different Reynolds numbers, one close to the critical Re for the apparatus $\mathrm{Re}_{\mathrm{C}}$, and one larger than $\mathrm{Re}_{\mathrm{C}}$ in order to investigate the influence of $\mathrm{Re}$ on some turbulent quantities that govern the mixing processes.
Before of each test, 1500 frame of the background were recorded, afterwards, the solution was released and, when a steady flow state was established, a minimum of 4000 independent samples was acquired

Table 1. Experiments run details.

\begin{tabular}{|c|c|c|c|c|c|c|}
\hline $\begin{array}{c}\text { Tes } \\
\text { ts }\end{array}$ & $\begin{array}{c}\Theta \\
{\left[{ }^{\circ}\right]}\end{array}$ & $\mathbf{R e}$ & $\begin{array}{c}\mathbf{Q} \\
{\left[\mathbf{m}^{3} / \mathbf{s}\right]}\end{array}$ & $\begin{array}{c}\Delta \rho \\
{\left[\mathrm{kg} / \mathbf{m}^{3}\right]}\end{array}$ & $\begin{array}{c}\rho \\
{\left[\mathrm{kg} / \mathbf{m}^{3}\right]}\end{array}$ & $\mathbf{F}$ \\
\hline 1 & 80 & 700 & $3 \times 10^{-6}$ & 6.5 & 1000 & 15 \\
\hline 2 & 65 & 700 & $3 \times 10^{-6}$ & 6.5 & 1000 & 15 \\
\hline 3 & 80 & 1500 & $6 \times 10^{-6}$ & 26 & 1000 & 15 \\
\hline 4 & 65 & 1500 & $6 \times 10^{-6}$ & 26 & 1000 & 15 \\
\hline
\end{tabular}

Due to the high range of velocities typical of this phenomenon (very high at the outlet and very low far from the outlet), two different frames were recorded, for each run, a first frame ("near field") close to the outlet, with a resolution of $2240 \times 864$ and a frequency of 800 $\mathrm{Hz}$, and a second frame ("far field") including the whole of interest, to study all the behaviour of the jet along its path, with a resolution o $2240 \times 1728$ and a frequency of $400 \mathrm{~Hz}$.

\subsection{Feature Tracking Velocimetry algorithm}

Velocity fields were obtained, from each couple of images, using a Feature Tracking Velocimetry algorithm. The idea of this technique is to compare windows only where the motion detection may be successful, so where there are high luminosity gradients.

Different from classical PIV, which obtain fields comparing windows of successive frame on a regular grid over the whole image, but also from Particle tracking Velocimetry (PTV), as there is no need to identify particle centroids. Therefore, this technique is suitable for measurements from a wide range of seeding density: from the high level typical of the PIV down to the low seeding which is characteristic of the PTV. The algorithm is also suitable in presence of non-homogeneous seeding density, for example between the jet and the receiving fluid, where the common techniques have significant errors, due to the non-homogeneous seeding at the boundary.

The procedure of analysis consist of:

- identification of the features using the Harris corner detection (a corner is a region with high luminosity gradients along the $\mathrm{x}$ and $\mathrm{y}$ direction) (Harris and Stephens, 1988 [37]);

- $\quad$ ordering the features according to their corneress (the value of the Harris formula), and choice the best features;

- selecting the first $\mathrm{N}$ features and computing displacement, comparing a window centred with the i-th feature $\left(\mathrm{W}_{\mathrm{t}}\right)$ with windows $\left(\mathrm{W}_{\mathrm{t}+1}\right)$ with a range of displacements, $\left(\mathrm{d}_{\mathrm{i}}, \mathrm{d}_{\mathrm{j}}\right)$ in the next frame; for each displacement a measure of the dissimilarity between $\mathrm{W}_{\mathrm{t}}$ and $\mathrm{W}_{\mathrm{t}+1}\left(\mathrm{~d}_{\mathrm{i}}, \mathrm{d}_{\mathrm{j}}\right)$; the 
measure of dissimilarity, $\mathrm{d}(\mathrm{di}, \mathrm{dj})$ is computed using the Lorentzian estimator.

- the displacement is chosen as the one minimizing the dissimilarity, computed using the Lorentzian estimator, and the velocity obtained as the displacements divided by the time shift between the two images;

- the samples are validated with an algorithm based on a Gaussian filtering of first neighbours (define by the Delaunay triangulation).

Under the hypothesis of ergodicity, the statistics of velocity are subsequently obtained by time averaging.

\section{Results}

\subsection{General behaviour of the negatively buoyant jets}

Mean velocity fields normalized by the maximum exit velocity, $U_{\max }$ are displayed in figures 4 and 5 for lower and higher Reynolds number, respectively. In each plot the dash-dot line represents the jet axis, defined as the locus of maximum velocities. As apparent from velocity fields, NBJs cover a very short initial distance, where they maintain a width similar to the diameter of the orifice, and after a length of few diameters their width grows due to the onset of the Kelvin-Helmholtz (KH) waves.
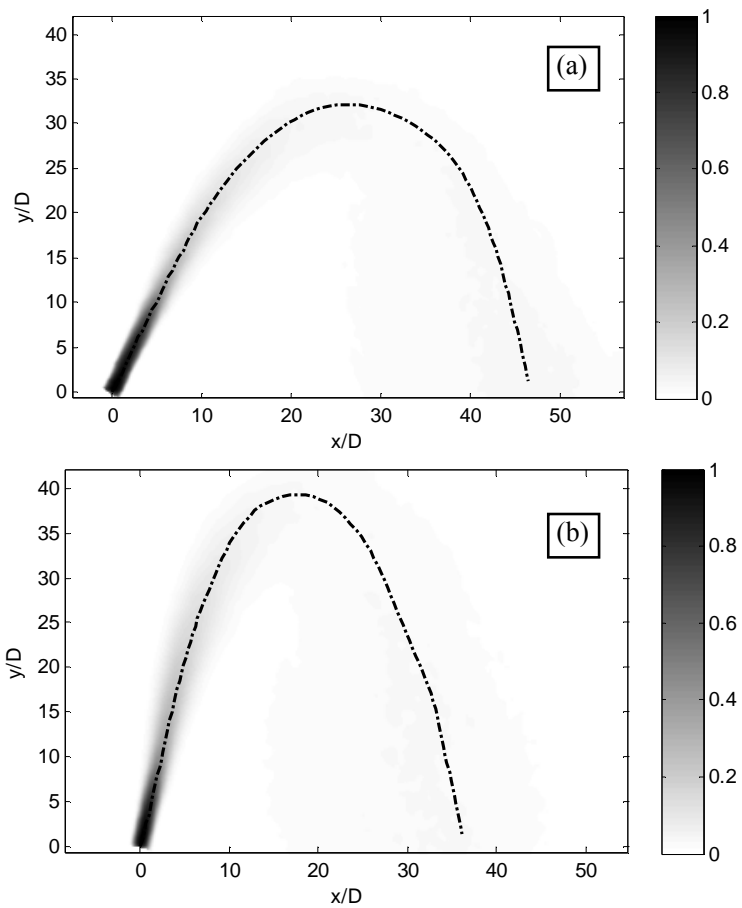

Fig. 4. (a) Map of the non-dimensional mean velocity $\mathrm{U} / \mathrm{U}_{\mathrm{MAX}}\left(\mathrm{U}_{\mathrm{MAX}}\right.$ is the maximum initial velocity), for a jet having $\mathrm{Fr}=15, \mathrm{Re}=700$ and inclination $80^{\circ}$. The dashdot line represent the jet axis. (b) Map of the nondimensional mean velocity $\mathrm{U} / \mathrm{U}_{\mathrm{MAX}}\left(\mathrm{U}_{\mathrm{MAX}}\right.$ is the maximum initial velocity), for a jet having $\mathrm{Fr}=15, \mathrm{Re}=$ 700 and inclination $65^{\circ}$. The dash-dot line represent the jet axis.
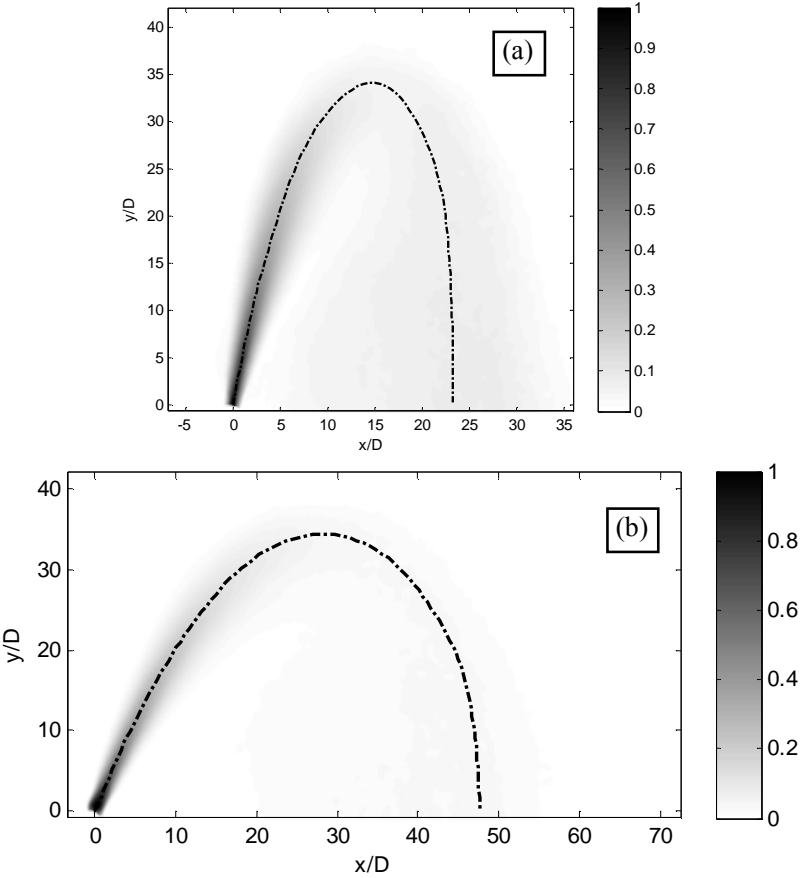

Fig. 5. (a) Map of the non-dimensional mean velocity $\mathrm{U} / \mathrm{U}_{\mathrm{MAX}}\left(\mathrm{U}_{\mathrm{MAX}}\right.$ is the maximum initial velocity), for a jet having $\mathrm{Fr}=15, \mathrm{Re}=1500$ and inclination $80^{\circ}$. The dashdot line represent the jet axis. (b) Map of the nondimensional mean velocity $\mathrm{U} / \mathrm{U}_{\mathrm{MAX}}\left(\mathrm{U}_{\mathrm{MAX}}\right.$ is the maximum initial velocity), for a jet having $\mathrm{Fr}=15, \mathrm{Re}=$ 1500 and inclination $65^{\circ}$. The dash-dot line represent the jet axis.

Figure 4 and 5 show that for higher Reynolds number the jet widens more that for low one, and is characterized by a higher dissipation, while fields corresponding to lower Reynolds numbers present more persistent jet cores that cover longer distance. Moreover, it is possible to notice an asymmetry of the velocity fields with respect to the jet axis between the upper and the lower part of the jet.

This behavior is better highlighted from velocity profiles orthogonal to the jet axis, computed at different nondimensional distances along the axis, $\mathrm{s} / \mathrm{D}$, and plotted in figures 6 and 7 after being normalized by their maximum velocity $\left(U_{c}\right)$. The mean velocity profiles computed near the orifice are symmetrical, and their shape is a typical bell-gaussian, this behavior persists until the initial momentum is relevant, but for higher s/D values, once the buoyancy forces become more relevant, this axisymmetry vanishes. Actually, at the lower boundary the $\mathrm{KH}$ waves are contrasted in their complete development by the buoyancy forces, and they are transformed into plumes propagating downwards, causing fat tails in the velocity profiles, while at the upper boundary the local stable stratification, permits to the $\mathrm{KH}$ waves to develop completely and the profile maintains a bell shape.

Comparing the two plots in the figure 4 and 5 , it is important to notice that the jet axis reaches almost the same height, this is due to the re-entrainment phenomenon, i.e. the mixing of the jet fluid with itself instead of being fed by the external fluid (Ferrari et al. 2010 [1]), this condition occurs for inclination exceeding $75^{\circ}$. 

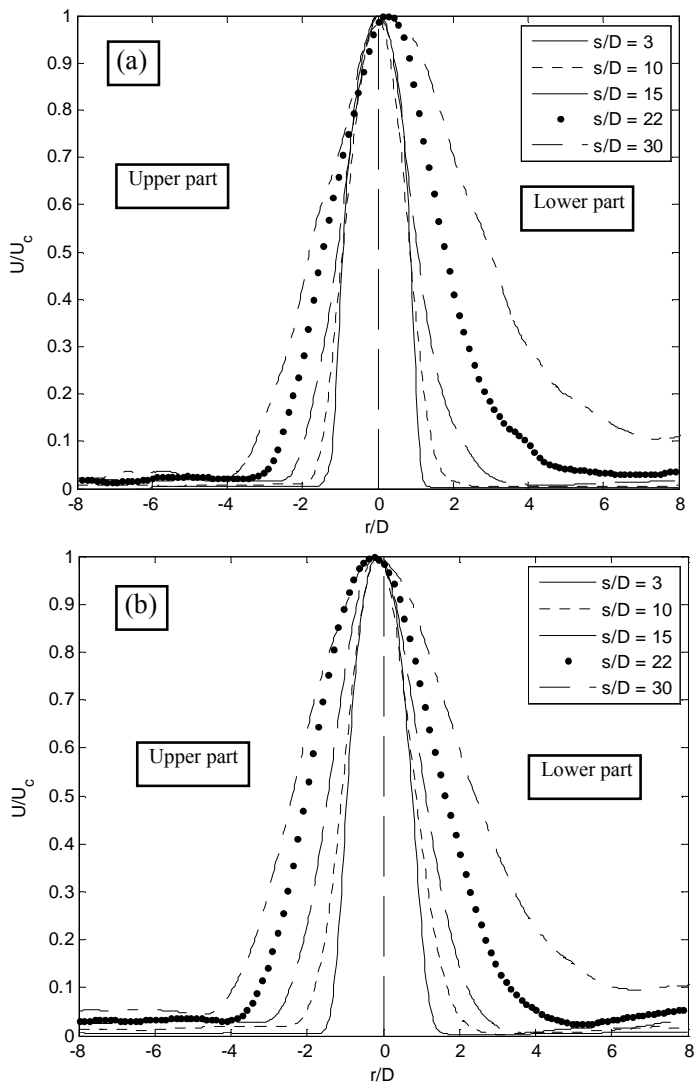

Fig. 6. (a) Velocity profiles orthogonal to the jet axis, for a jet having $\mathrm{Fr}=15, \mathrm{Re}=700$ and inclination $80^{\circ}$, normalized by the maximum axial velocity. (b) Velocity profiles orthogonal to the jet axis, for a jet having $\mathrm{Fr}=$ $15, \operatorname{Re}=700$ and inclination $65^{\circ}$, normalized by the maximum axial velocity.

Figure 8 shows a zoom in the region near to the diffuser, of the mean velocity field obtained for a release angle of $80^{\circ}$, in presence of rentrainment: one can notice the velocity recirculation pattern, which causes the lower part of the jet, near the orifice, to be fed by the descending part of the jet, and hence a lower dilution.

Looking the jet axis, is evident how the geometrical characteristic of the jet are independent on the Reynolds number, so the jet reaches the same height in the two cases, and also covers the same distance, but the dissipation of velocity is lower, presents a compact core of velocity until $l_{M}\left(l_{M}\right.$ for this jets is 14.4), afterwards the dissipation of velocity is higher than the one obtained for the fully turbulent jet.

This is evident in the figure 9, where is shown the velocity of the jet axis, in the four condition simulated, in the image (a) for inclination of $65^{\circ}$, and in (b) for an angle of $80^{\circ}$. The centerline velocity decays, obtained from experimental results, were compared to the law valid for simple jets $U_{m} \approx 7 \cdot M_{0}{ }^{1 / 2} \cdot s^{-1}$ (List et al. 1979 [34]).

For NBJs, simulated in this work, the centreline velocity decay, follows closely, the one for simple jets, up to $l_{\mathrm{M}}$.

Actually in case of lower Reynolds, there is a persistent velocity core of about 7 diameters, where the decay is lower, but beyond this value the velocity decay is faster than the one observed for simple jets.
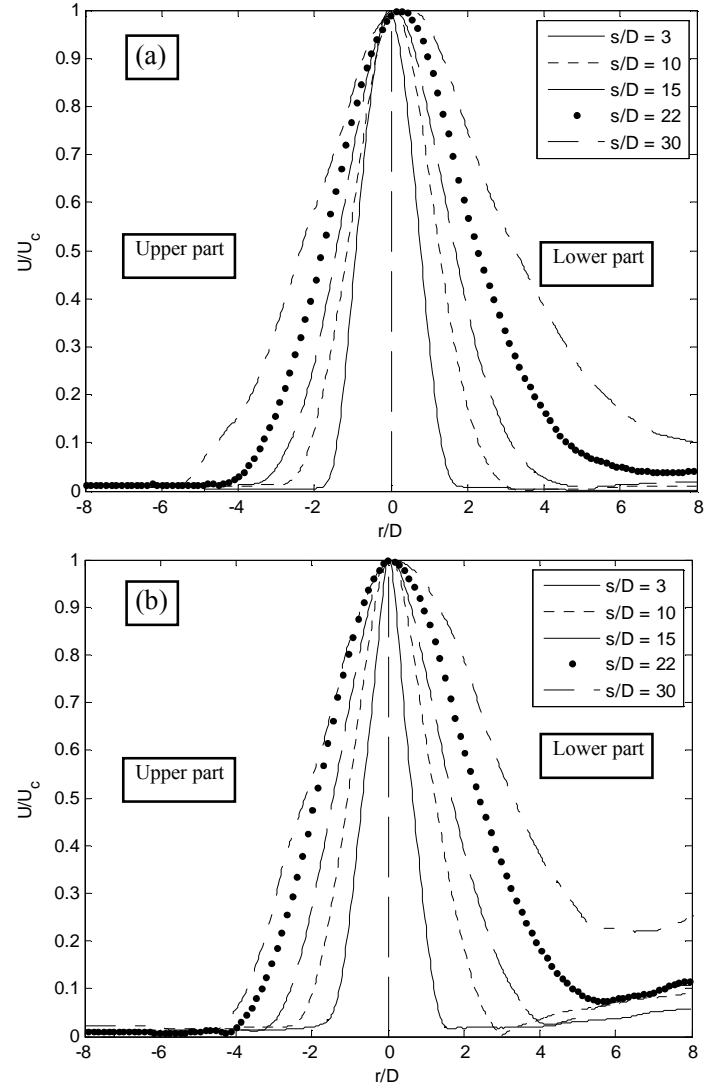

Fig. 7. (a) Velocity profiles orthogonal to the jet axis, for a jet having $\mathrm{Fr}=15, \mathrm{Re}=1500$ and inclination $80^{\circ}$, normalized by the maximum axial velocity. (b) Velocity profiles orthogonal to the jet axis, for a jet having $\mathrm{Fr}=15$, $\operatorname{Re}=1500$ and inclination $65^{\circ}$, normalized by the maximum axial velocity.

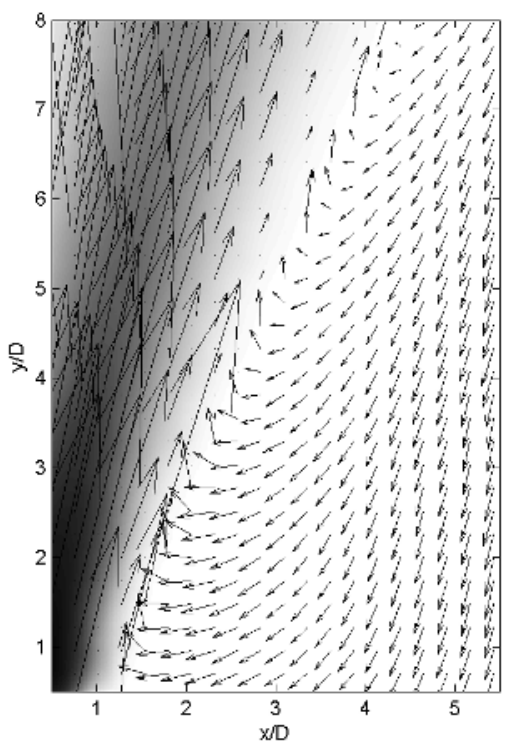

Fig. 8. Detail of the map of the non-dimensional mean velocity $\mathrm{U} / \mathrm{U}_{\mathrm{MAX}}$, in presence of re-entrainment, for a jet with $\mathrm{Fr}=15, \mathrm{Re}=1500$ and inclination $80^{\circ}$. 

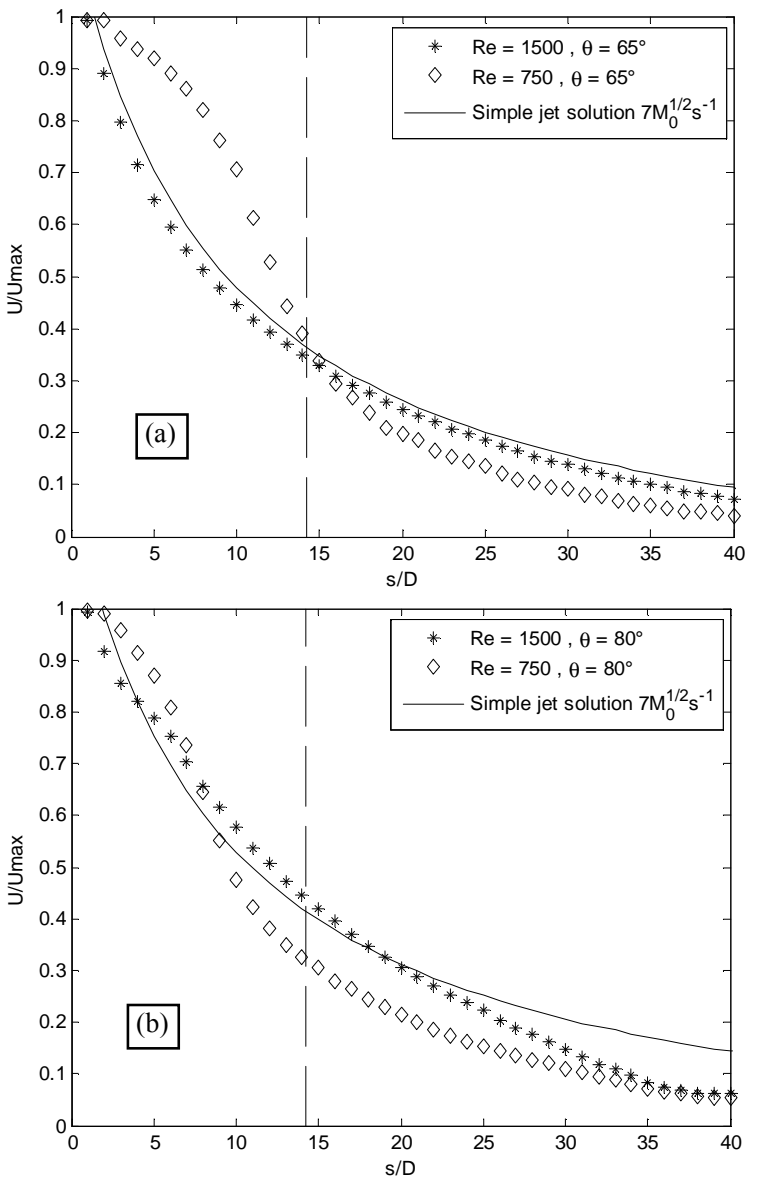

Fig. 9. (a) Jet centreline velocity decay, for an angle of $65^{\circ}$ and $\mathrm{Fr}=15$, the starred line having a $\mathrm{Re}=1500$ (fully turbulent), the squares $\mathrm{Re}=750$ (critical), the continuous line is the equation of the velocity decay for simple jet (List et al. 1979), the dotted line is $1_{M}$ (before $l_{M}$ is valid the velocity decay law of List). (b) Jet centreline velocity decay, for an angle of $80^{\circ}$ and $\mathrm{Fr}=$ 15 , the starred line $\operatorname{Re}=1500$, the squares $\operatorname{Re}=750$, the continuous line is the equation for simple jet, the dotted line is $1_{\mathrm{M}}$.

\subsection{Turbulence quantities}

\subsubsection{Turbulent Kinetic Energy}

The Turbulent Kinetic Energy (TKE), can be defined as follows:

$$
T K E=\frac{1}{2}\left(\overline{u_{x}^{\prime 2}}+\overline{u_{x}^{\prime 2}}\right)
$$

where $\mathrm{u}_{\mathrm{x}}$ and $\mathrm{u}_{\mathrm{y}}$ are the velocity fluctuations along the $\mathrm{x}$ and $y$ axis, while the overbars represent an ensamble average.

Figure 10 shows a map of the mean TKE field computed for the two different Reynolds number analyzed.

TKE fields present low values in the central jet region, and two high value regions at the jet boundaries, where turbulence is originated by the buoyant and the mechanical generated eddies. The highest maximum is in the upper boundary, where the TKE is countered by the buoyancy acting in the opposite direction of the momentum, and allows the KH waves to develop completely. This condition is evident until $\mathrm{l}_{\mathrm{M}}$, is reached, where the buoyancy prevails, the two TKE peaks disappear, and the jet becomes fully turbulent.
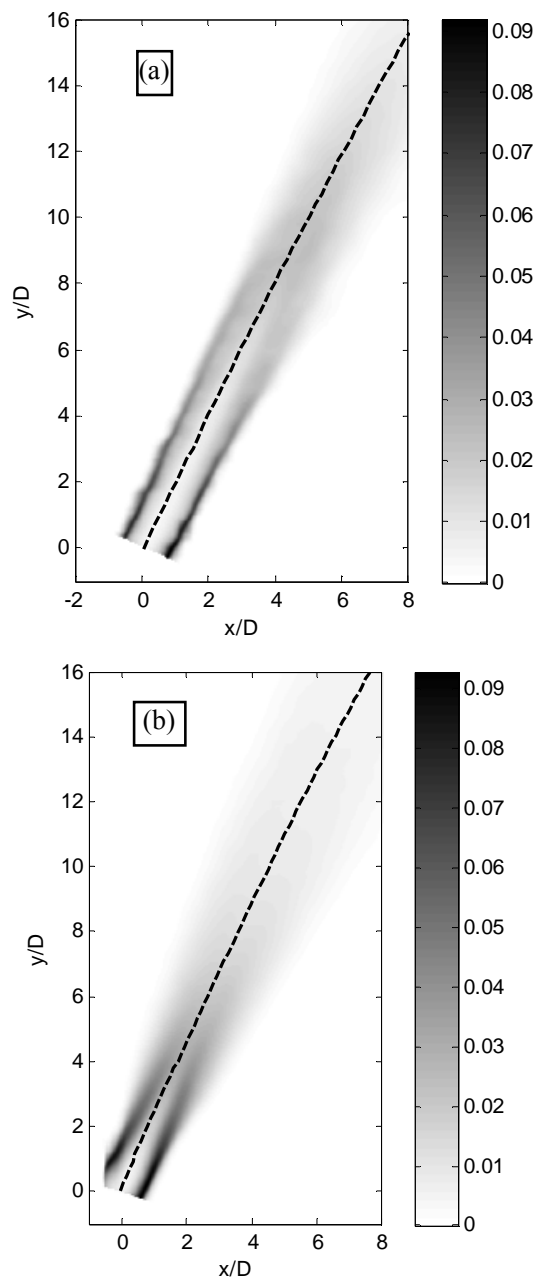

Fig. 10. (a) Map of the non-dimensional mean turbulent kinetic energy (TKE), normalized with $\mathrm{U}^{2}{ }_{\text {MAX, }}$, for a jet having $\mathrm{Fr}=15, \mathrm{Re}=700$ and inclination $65^{\circ}$. The dashdot line represent the jet axis (defined as the locus of maximum intensity velocity). (b) Map of the nondimensional mean turbulent kinetic energy (TKE), normalized with $\mathrm{U}^{2}{ }_{\mathrm{MAX}}$, for $\mathrm{Fr}=15, \mathrm{Re}=1500$ and inclination $65^{\circ}$.

The non turbulent jet core, characterized by small TKE values, further persists along the jet axis in case of low Reynolds number, while for high Reynolds number the transition to turbulence occurs nearer to the diffuser.

Figure 11 shows the TKE profiles computed at different distances $\mathrm{s} / \mathrm{D}$, the lack of symmetry between the lower and the upper part of the jet is apparent. This nonaxialsymmetric behaviour implies that the usual integral equations for simple jets and plumes, developed under the hypothesis of axisymmetry, cannot provide to precise results. 

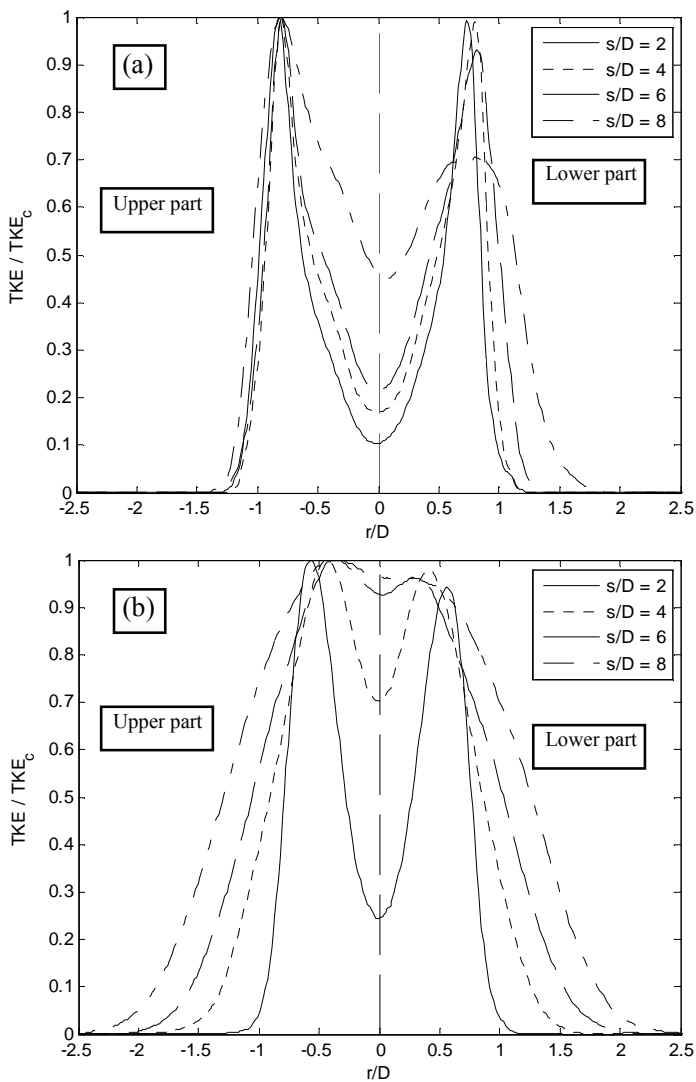

Fig. 11. (a) TKE profiles orthogonal to the jet axis, of a jet having $\mathrm{Fr}=15, \mathrm{Re}=700$ and inclination $65^{\circ}$. (b) TKE profiles orthogonal to the jet axis, of a jet having $\mathrm{Fr}=15$, $\operatorname{Re}=1500$ and inclination $65^{\circ}$.

\subsubsection{Maximum Reynolds Stresses}

The generic component of the Reynolds stress tensor can be defined as:

$$
\tau_{R_{i j}}=\rho \overline{u_{i}^{\prime} u_{j}^{\prime}}
$$

where $\rho$ is the density of the flow, $u_{i}^{\prime}, u_{j}^{\prime}$ represent the velocity fluctuations for $\mathrm{i}$ and $\mathrm{j}$ components, while $i$ and $j$ may stand for $x$ and $y$. It can be demonstrated (Marvern et al. 1977 [38]) that the maximum Reynold stress, whose direction is not aligned with the coordinate system, can be computed in terms of the measured velocity components, and expressed as:

$$
\tau_{R \max }=\rho \sqrt{\frac{1}{4}\left(\overline{u_{y}^{2}}-\overline{u_{x}^{2}}\right)^{2}+\left(\overline{u_{x}^{\prime} u_{y}^{\prime}}\right)^{2}}
$$

Figure 12 shows the map of the maximum Reynolds stress for experiments performed with a release angle of $65^{\circ}$. They display a similar behavior to the one obtained for TKE fields. Low values are observed in the central jet region, while two high value regions are present at the jet borders, with the maximum in the upper boundary, where the Kelvin - Helmholtz structures develop upwards, and rapidly they break, due to the buoyancy contrasting their growth.
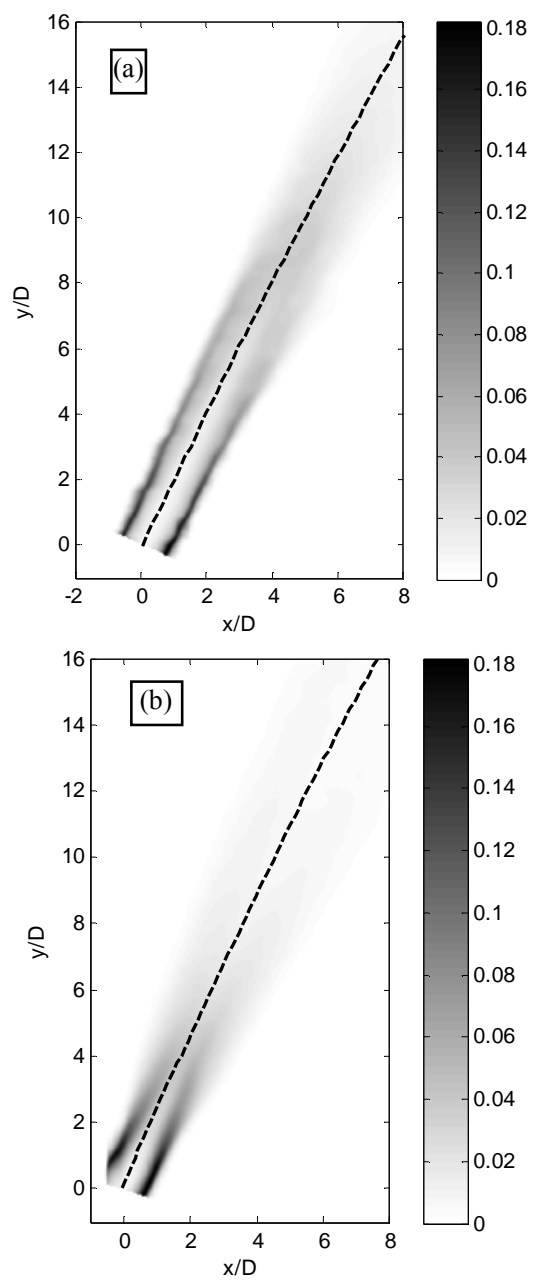

Fig. 12. (a) Map of the non-dimensional maximum turbulent Reynolds stresses $\left(t_{R}\right)$, normalized with $\mathrm{U}^{2}{ }_{\mathrm{MAX}}$, for a jet having $\mathrm{Fr}=15, \operatorname{Re}=700$ and inclination $65^{\circ}$. The dash - dot line represent the jet axis (defined as the locus of maximum intensity velocity). (b) Map of the non-dimensional maximum turbulent Reynolds stresses $\left(t_{R}\right)$, normalized with $U^{2}{ }_{M A X}$, for a jet having $F r=15, \operatorname{Re}$ $=1500$ and inclination $65^{\circ}$, normalized by $\mathrm{U}^{2} \mathrm{MAX}$

It is apparent how for both Reynolds number, the $\mathrm{KH}$ waves originate at the outlet, however, their structure is very different and for high Re, the momentum, allow the waves to develop better, causing an higher mixing, but the dissipation of momentum is also higher, and the maximum decays rapidly, otherwise, with a low Reynolds this waves are smaller, but more persistent.

This behavior is better clarified from the analysis of the non-dimensional profiles computed at different sections on the jet axis and displayed in figure 13. 

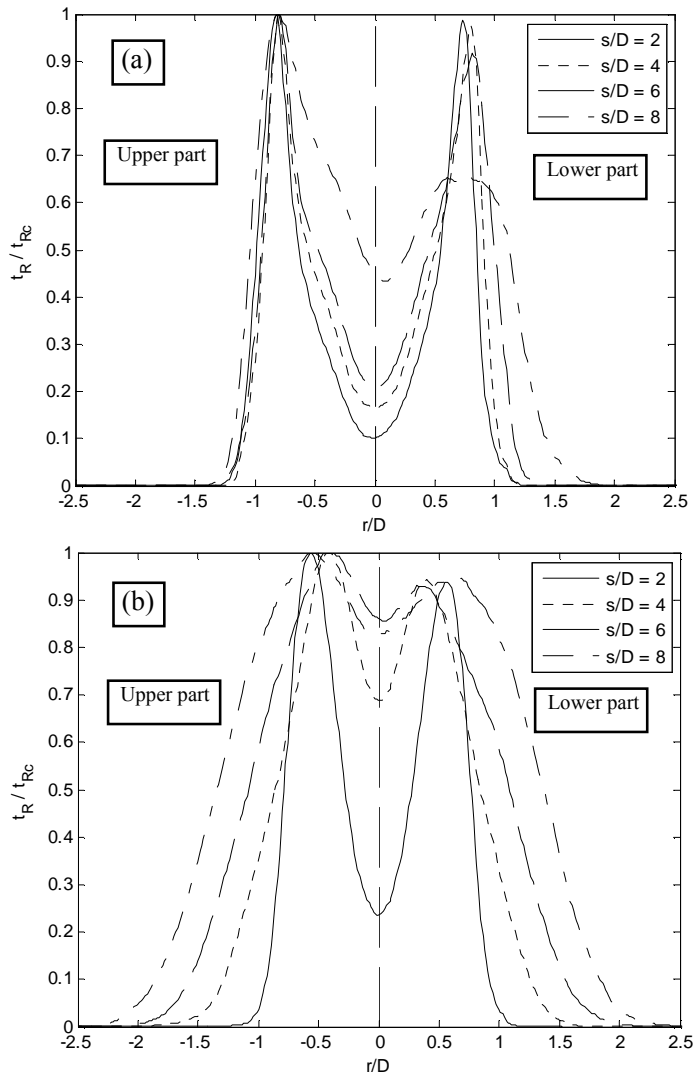

Fig. 13. (a) $t_{R}$ profiles orthogonal to the jet axis, for a jet having $\mathrm{Fr}=15, \mathrm{Re}=700$ and inclination $65^{\circ}$, normalized by $\mathrm{U}^{2}$ MAX (non-dimensional axis coordinates $\mathrm{s} / \mathrm{D}$ are shown in legend). (b) (a) $t_{R}$ profiles orthogonal to the jet axis, for a jet having $\mathrm{Fr}=15, \mathrm{Re}=1500$ and inclination $65^{\circ}$, normalized by $\mathrm{U}^{2}$ MAX

\section{Conclusions}

The behaviour of Negatively Buoyant Jets, released from a sharp-edged orifice, was investigating using an Feature Tracking Velocimetry, an original and efficient image analysis technique Mean velocity fields and second order statistics were used to characterize the behaviour of this phenomenon and to assess its dependence on some jet parameters. Observed lack of axial symmetric behaviour points out that caution must be observed when using integral solutions or applying numerical codes based on the hypothesis of axial symmetry.

\section{Acknowledgements}

The authors are grateful to Antonio Mascia for his valuable contribution to the experimental work, and to Regione Sardegna, for the economical support:

- Legge Regionale 7 agosto 2007, n. 7: "Promozione della ricerca scientifica e dell'innovazione tecnologica in Sardegna"

- $\quad$ Phd fellowship: P.O.R. Sardegna Fse 2007-2013

\section{References}

1. S. Ferrari, G. Querzoli, J. Hydraulic Research, 48, 5 (2010)
2. A. Cipollina, A. Brucato, F. Grisafi, S. Nicosia, J. Hydraulic Eng., 131, 11 (2005)

3. G. A. Kikkert, M.J. Davidson, R.I. Nokes, J. Hydraulic Eng., 133, 5 (2007)

4. T.N. McLellan, R. Randall, J. Water, Port, Coast, Ocean Eng., 112, 2 (1986)

5. R.C.Y. Koh, M.S. Isaacson, N.H. Brooks, J. Hydraulic Eng., 109, 2 (1975)

6. W.D. Baines, J.S. Turner, I.H. Campbell, J.Fluid Mech. 212 (1989)

7. McClimans et al. 2000 McClimans, T., Eidnes, G. Proc. $5^{\text {th }}$ Intl. Symp. Stratified Flows, Vancouver (2000)

8. Lane-Serff, G.F., Linden, P.F., Hillel, M. J. Hazardous Mat. 33 (1993).

9. J.S. Turner, I.H. Campbell, Earth-Science Reviews, 23 (1986)

10. I.H. Campbell, J.S. Turner (1989). J. Petrol. 30 (1989)

11. Kaminski and Carazzo 2004 [11]). E. Kaminski, S. Tait, G. Carazzo J. Fluid Mech. 526 (2005)

12. D. Goldman Y. Jaluria J. Fluid Mechanics, 166 (1986)

13. W.D. Baines, J.S. Turner, I.H. Campbell, J. Fluid Mech 212 (1990)

14. H. Zhang, R.E. Baddour, J. Hydraulic Eng., 124, 5 (1998).

15. M.J. Davidson, K.L. Pun, I.R. Wood, J. Hydraulic Res. 40, 4 (2002)

16. N.B. Kaye, G.R. Hunt, J. Fluid Mechanics, 558, (2006)

17. C. H. Bosanquet, G. Horn, M.W. Thring, 1961. Proceedings of the Royal Society of London, Series A., 263, 1314 (1961)

18. M.A. Zeitoun, R.O. Reid, W.F. McHilhenny, T.M. Mitchell, Res. and Devel. Progress Rep. 804. Office of Saline Water, U.S. Dept. of the Interior, Washington DC. (1972)

19. P.J.W. Roberts, G. Toms, J. Hydraulic Eng. 113, 3 (1987)

20. P.J.W. Roberts, A. Ferrier, G. Daviero, J. Hydraulic Eng. 123, 8 (1997)

21. L.J. Bloomfield, R.C. Kerr, J. Fluid Mechanics 451, (2002)

22. G.A. Kikkert, M.J. Davidson, R.I. Nokes, J. Hydraulic Eng. 133, 5 (2007)

23. G.A. Kikkert, M.J. Davidson, R.I. Nokes, J. Hydraulic Research. 48, 3 (2010)

24. I.G. Papakonstantis, G.C. Christodoulou, P.N. Papanicolaou, J. Hydraulic Res. 49, 1 (2011a)

25. I.G. Papakonstantis, G.C. Christodoulou, P.N. Papanicolaou, J. Hydraulic Res. 49, 1 (2011b)

26. S. Ferrari, G. Querzoli, In: Avanzini, C. (Ed.): Proceedings of MWWD \& IEMES 2004, Catania (Italy), 27/9-2/10 2004

27. J. Mi, G.J. Nathan, D.S. Nobes, J. Fluid Eng. ASME 123, 4 (2001)

28. J. Mi, P. Kalt, G.J. Nathan, C.Y. Wong, Exp. Fluids 42, 4 (2007)

29. Ferrari S., Querzoli G., $7^{\text {th }}$ Int. Symp. on Stratified Flows, Rome, Italy, August 22 - 26, (2011) 
30. A. Cenedese, G. Querzoli, J. Hydraul. Res, 43, 1 (2005)

31. Lai C.C.K., Lee J.H.W., J. Hydro-environment Res. $6(2012)$

32. D.D. Shao, A.W.K. Law, Environmental Fluid Mechanics, 10, 5 (2010)

33. D.D. Shao, A.W.K. Law, J. Hydro-environment Res. $5(2011)$

34. List, EJ: In: Fisher, HB - List, EJ - Koh, RCY Imberger, $\mathrm{J}$ - Brooks, $\mathrm{NH}$ (Eds): Mixing in inland and coastal water, New York, USA, Academic Press, (1979)

35. S.J. Wright, D.R. Wong, K.E. Zimmerman, R.B. Wallace J. Hydraulics Div. ASCE 108 (HY4) (1982)

36. C. Avanzini, Proc. 4th Intl. Conf Marine Waste Water Disposal and Marine Environment \& 2nd Intl. Exhibition Materials, Equipment and Services for Coastal Waste Water Treatment Plants, Outfalls and Sealines Antalya, Turkey, Mem Ajans, Istanbul (2006)

37. C. Harris, M. Sthephens, In : Procedings of the 4th Aivey Vision Conference, pp 141-151 (1988)

38. L.E. Malvern, Prentice-Hall, Englewood Clils, NJ (1977) 